\title{
Particle Dark Matter in the galactic halo: Results from DAMA/LIBRA
}

R. Bernabei $\left({ }^{1}\right)$, P. Belli $\left({ }^{1}\right)$, F. Montecchia $\left({ }^{1}\right)$, F. Nozzoli $\left({ }^{1}\right)$, F. Cappella $\left({ }^{2}\right)$, A. D'Angelo $\left({ }^{2}\right)$, A. Incicchitti $\left({ }^{2}\right)$, D. Prosperi $\left({ }^{2}\right)$, R. Cerulli $\left({ }^{3}\right)$, C. J. Dai $\left({ }^{4}\right)$, H. L. He $\left({ }^{4}\right)$, H. H. Kuang $\left({ }^{4}\right)$, X. H. Ma $\left({ }^{4}\right)$, X. D. Sheng $\left({ }^{4}\right)$ and Z. P. $\mathrm{Ye}\left({ }^{4}\right)$

$\left({ }^{1}\right)$ Dipartimento di Fisica, Università di Roma "Tor Vergata" INFN, Sezione di Roma "Tor Vergata" - I-00133 Rome, Italy

$\left(^{2}\right)$ Dipartimento di Fisica, Università di Roma "La Sapienza" INFN, Sezione di Roma - I-00185 Rome, Italy

$\left(^{3}\right)$ INFN, Laboratori Nazionali del Gran Sasso - Assergi, Italy

(4) IHEP, Chinese Academy - P.O. Box 918/3, Beijing 100039, China

(ricevuto il 19 Settembre 2009; pubblicato online il 20 Novembre 2009)

Summary. - The DAMA/LIBRA experiment at the Gran Sasso National Laboratory of the INFN has confirmed the model-independent evidence for Dark Matter (DM) particles in the galactic halo on the basis of the DM annual modulation signature. Considering these data together with those of the former DAMA/NaI a confidence level of $8.2 \sigma$ is achieved for a cumulative exposure of 0.82 ton $\times y$. Recently a first upgrading of the set-up has been carried out and a second one is in preparation; this latter one has the main aim to decrease the energy threshold of the experiment. Moreover, investigations on other rare processes are also in progress.

PACS 29.40.Mc - Scintillation detectors.

PACS 95.30. Cq - Elementary particle processes.

PACS 95.35.+d - Dark matter (stellar, interstellar, galactic, and cosmological).

DAMA is an observatory for rare processes and it is in operation deep underground at the Gran Sasso National Laboratory of the INFN. It is mainly based on the development and use of low background scintillators. The main experimental set-ups are: i) DAMA/NaI [1,2]; ii) DAMA/LXe [3,4]; iii) DAMA/R\&D [5]; iv) DAMA/Ge and LNGS Ge facilities [6]; v) the new second generation DAMA/LIBRA set-up $(\simeq 250 \mathrm{~kg}$ highly radiopure $\mathrm{NaI}(\mathrm{Tl})$ ) [7-9]. Profiting of the low background features of these setups, many rare processes are investigated obtaining often competitive results. In particular, DAMA/LIBRA is further investigating the presence of DM particles in the galactic halo by exploiting the model-independent DM annual modulation signature. This signature - originally suggested in the middle of ' 80 in ref. [10] - exploits the effect of the Earth revolution around the Sun on the number of events induced by DM particles in the detectors. In fact, as a consequence of its annual revolution, the Earth should be 
crossed by a larger flux of DM particles around $\sim 2$ June (when its rotational velocity is summed to the one of the solar system with respect to the Galaxy) and by a smaller one around $\sim 2$ December (when the two velocities are subtracted). This offers an efficient model-independent signature and allows to test large intervals of cross-sections and halo densities. The DM annual modulation signature is very distinctive since the corresponding signal must simultaneously satisfy all the following requirements: the rate must contain a component modulated according to a cosine function 1) with one year period 2) and a phase that peaks around $\simeq 2$ nd June (3); this modulation must only be found in a well-defined low energy range, where DM particle induced events can be present (4); it must apply only to those events in which just one detector of many actually "fires" (single-hit events), since the DM particle multi-interaction probability is negligible (5); the modulation amplitude in the region of maximal sensitivity must be $\lesssim 7 \%$ for usually adopted halo distributions (6), but it can be larger in case of some possible scenarios such as, e.g., those in refs. [11,12]. Only systematic effects or side reactions able to fulfil these 6 requirements and to account for the whole observed modulation amplitude might mimic this signature $\left({ }^{1}\right)$; thus, no other effect investigated so far in the field of rare processes offers a so stringent and unambiguous signature.

Detailed descriptions of DAMA/NaI [13-16] and of DAMA/LIBRA [7] performances have been published. The DAMA/NaI experiment collected an exposure of 0.29 ton $\times y$ over 7 annual cycles, while DAMA/LIBRA has released so far an exposure of 0.53 ton $\times y$ collected over 4 annual cycles; thus, the total exposure of the two experiments is 0.82 ton $\times y$, which is orders of magnitude larger than the exposure typically collected in the field. Several model-independent analyses have been performed in order to investigate the DM annual modulation signature [8]. In fig. 1 top there is shown the time behaviour of the experimental (2-6) keV residual rates for single-hit events collected by DAMA/NaI and by DAMA/LIBRA. The superimposed curve represents the cosinusoidal functions: $A \cos \omega\left(t-t_{0}\right)$ with $T=\frac{2 \pi}{\omega}=1 \mathrm{y}$ and phase $t_{0}=152.5$ day (June 2 nd), while the modulation amplitudes, $A$, is the best-fit value obtained over the DAMA/NaI and DAMA/LIBRA data. When the period and the phase parameters are released in the fit, values well compatible with those expected for a DM particle induced effect are obtained [8]. The same data of fig. 1 have also been investigated by a Fourier analysis [8]. For all the performed analyses and for details see ref. [8]. In particular, a relevant investigation has been performed by applying the same hardware and software procedures, used to acquire and to analyse the single-hit events, to the multiple-hits ones. In fact, since the probability that a DM particle interacts in more than one detector is negligible, a DM signal can be present just in the single-hit residual rate. Thus, this allows the verification of the background behaviour in the same energy interval where the positive effect is observed. In particular, while a clear modulation is present in the single-hit events in the $2-6 \mathrm{keV}$ energy interval, the modulation amplitude for the multiple-hits ones in the same energy interval is well compatible with zero [8] (see fig. 1 bottom). Similar results were previously obtained also for the DAMA/NaI case [16]. Thus, again evidence of annual modulation with proper features, as required by the DM annual mod-

$\left({ }^{1}\right)$ It is worth noting that the DM annual modulation is not-as often naively said - a "seasonal" variation and it is not a "winter-summer" effect. In fact, the DM annual modulation is not related to the relative Sun position, but it is related to the Earth velocity in the galactic frame. Moreover, the phase of the DM annual modulation (roughly 2nd June) is well different than those of physical quantities (such as temperature of atmosphere, pressure, other meteorological parameters, cosmic rays flux, ... ) instead correlated with seasons. 

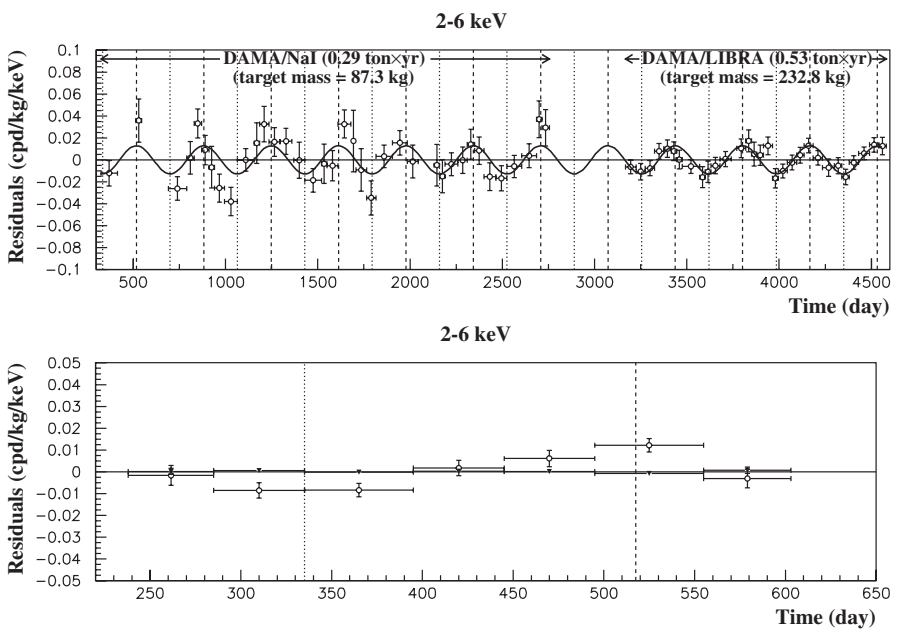

Fig. 1. - Top: experimental model-independent residual rate of the single-hit scintillation events, measured by DAMA/NaI and DAMA/LIBRA in the (2-6) keV energy intervals as a function of the time. The zero of the time scale is January 1st of the first year of data taking of the former DAMA/NaI experiment. The superimposed curve is the cosinusoidal functions with 1 year period, June 2nd phase and fitted amplitude $(0.0129 \pm 0.0016) \mathrm{cpd} / \mathrm{kg} / \mathrm{keV}$. The dashed vertical lines correspond to the maximum of the signal (June 2nd), while the dotted vertical lines correspond to the minimum. Bottom: experimental model-independent residual rates of the (2-6) keV single-hit events (open circles) (class of events to which DM events belong) and of the (2-6) keV multiple-hits events (filled triangles) (class of events to which DM events do not belong), measured in the four DAMA/LIBRA annual cycles (as collected in a single cycle; the initial time of the scale is taken on August 7th). The same identical hardware and the same identical software procedures have been applied in both cases. In the plots the experimental points present the errors as vertical bars and the associated time bin width as horizontal bars. For details see [8].

ulation signature, is present in the single-hit residual rate (events class to which the DM particle induced events belong), while it is absent in the multiple-hits one (event class to which only background events belong). Since the same identical hardware and the same identical software procedures have been used to analyse the two classes of events, the obtained result offers an additional strong support for the presence of a DM particle component in the galactic halo further excluding any side effect either from hardware or from software procedures or from background.

All the performed analyses [8] confirm that a modulation amplitude is present in the lower energy intervals with the period and the phase in agreement with those expected for DM induced signals; in addition, the observed annual modulation fulfills all the requirements of the DM signature.

As previously done for the case of DAMA/NaI [14-16], careful investigations on the absence of any significant effect from systematics or side reactions have been quantitatively carried out also for DAMA/LIBRA; it is reported in details in ref. [8]. No systematics or side reactions able to account for the measured modulation amplitude and to contemporaneously satisfy all the requirements of the signature have been found or suggested by anyone over more than a decade.

For all details and for discussions see ref. [8] and references quoted therein. 
During September 2008 the first upgrading of the DAMA/LIBRA set-up has been realized by performing a new optimization of some PMTs and HVs, by replacing the transient digitizers with new ones and by installing a new DAQ system with optical readout. Moreover, mainly in order to lower the energy threshold of the experiment, the replacement of all the PMTs with new ones having higher quantum efficiency is planned; this will also improve other significant experimental aspects. The increasing of the exposure and the hardware improvements will allow to further deeply investigate open aspects in the DM field and, also, second-order effects.

Finally, other processes are also investigated by DAMA/LIBRA; in particular, recently new results on the search for possible processes violating the Pauli-ExclusionPrinciple in sodium and in iodine have been presented in ref. [9].

\section{REFERENCES}

[1] Bernabei R. et al., Phys. Lett. B, 389 (1996) 757; 424 (1998) 195; 450 (1999) 448; Belli P. et al., Phys. Rev. D, 61 (2000) 023512; Bernabei R. et al., Phys. Lett. B, 480 (2000) 23; 509 (2001) 197; Eur. Phys. J. C, 23 (2002) 61; Belli P. et al., Phys. Rev. D, 66 (2002) 043503; Bernabei R. et al., Riv. Nuovo Cimento, 26, no. 1 (2003) 1; Int. J. Mod. Phys. D, 13 (2004) 2127; Int. J. Mod. Phys. A, 21 (2006) 1445; Eur. Phys. J. C, 47 (2006) 263; Int. J. Mod. Phys. A, 22 (2007) 3155; Eur. Phys. J. C, 53 (2008) 205; Phys. Rev. D, 77 (2008) 023506; Mod. Phys. Lett. A, 23 (2008) 2125.

[2] Bernabei R. et al., Phys. Lett. B, 408 (1997) 439; Phys. Rev. Lett., 83 (1999) 4918; Nuovo Cimento A, 112 (1999) 1541; Phys. Lett. B, 515 (2001) 6; Eur. Phys. J. A, 23 (2005) 7; 24 (2005) 51; Belli P. et al., Phys. Lett. B, 460 (1999) 236; Phys. Rev. C, 60 (1999) 065501; Cappella F. et al., Eur. Phys. J.-direct C, 14 (2002) 1.

[3] Belli P. et al., Astropart. Phys., 5 (1996) 217; Nuovo Cimento C, 19 (1996) 537; Phys. Lett. B, 387 (1996) 222; 389 (1996) 783 (Erratum); 465 (1999) 315; Phys. Rev. D, 61 (2000) 117301; Bernabei R. et al., New J. Phys., 2 (2000) 15.1; Phys. Lett. B, 493 (2000) 12; Nucl. Instrum. Methods A, 482 (2002) 728; Eur. Phys. J. direct C, 11 (2001) 1; Phys. Lett. B, 527 (2002) 182; 546 (2002) 23; in the volume Beyond the Desert 2003 (Springer, Berlin) 2003, 365; Eur. Phys. J. A, 27 (2006) 35.

[4] Bernabei R. et al., Phys. Lett. B, 436 (1998) 379.

[5] Bernabei R. et al., Astropart. Phys., 7 (1997) 73; Nuovo Cimento A, 110 (1997) 189; Belli P. et al., Astropart. Phys., 10 (1999) 115; Nucl. Phys. B, 563 (1999) 97; Bernabei R. et al., Nucl. Phys. A, 705 (2002) 29; Belli P. et al., Nucl. Instrum. Methods A, 498 (2003) 352; Cerulli R. et al., Nucl. Instrum. Methods A, 525 (2004) 535; Bernabei R. et al., Nucl. Instrum. Methods A, 555 (2005) 270; Ukr. J. Phys., 51 (2006) 1037; BeLli P. et al., Nucl. Phys. A, 789 (2007) 15; Phys. Rev. C, 76 (2007) 064603; Phys. Lett. B, 658 (2008) 193; Eur. Phys. J. A, 36 (2008) 167; Nucl. Phys. A, 826 (2009) 256.

[6] Belli P. et al., Nucl. Instrum. Methods A, 572 (2007) 734; Nucl. Phys. A, 806 (2008) 388; 824 (2009) 101.

[7] Bernabei R. et al., Nucl. Instrum. Methods A, 592 (2008) 297.

[8] Bernabei R. et al., Eur. Phys. J. C, 56 (2008) 333.

[9] Bernabei R. et al., Eur. Phys. J. C, 62 (2009) 327.

[10] Drukier K. A. et al., Phys. Rev. D, 33 (1986) 3495; Freese K. et al., Phys. Rev. D, 37 (1988) 3388

[11] Smith D. and Weiner N., Phys. Rev. D, 64 (2001) 043502; Tucker-Smith D. and Weiner N., Phys. Rev. D, 72 (2005) 063509.

[12] Freese K. et al., astro-ph/0309279; Phys. Rev. Lett., 92 (2004) 11301.

[13] Bernabei R. et al., Nuovo Cimento A, 112 (1999) 545.

[14] Bernabei R. et al., Eur. Phys. J. C, 18 (2000) 283.

[15] Bernabei R. et al., Riv. Nuovo Cimento, 26, no. 1 (2003) 1.

[16] Bernabei R. et al., Int. J. Mod. Phys. D, 13 (2004) 2127. 